\title{
Clinical Implications from Recent Trials and New Guidelines for Stroke Prevention in Atrial Fibrillation
}

\author{
Andreas Müssigbrodt and Gerhard Hindricks
}

Physicians, Department of Electrophysiology, Heart Center, Leipzig University, Leipzig, Germany

\begin{abstract}
After the release of the 2010 guidelines of the European Society of Cardiology (ESC) and the 2011 update of the guidelines of the American Heart Association/American College of Cardiology (AHA/ACC), new evidence has emerged that requires adaption of current clinical practice in the treatment of atrial fibrillation (AF). In 2011, results were published from two central AF trials concerning prevention of stroke in patients with AF: the Rivaroxaban Once daily oral direct factor Xa inhibition Compared with vitamin $\mathrm{K}$ antagonist for prevention of stroke and Embolism Trial in Atrial Fibrillation (ROCKET-AF) and the Apixaban for Reduction in Stroke and Other Thromboembolic Events in Atrial Fibrillation (ARISTOTLE) study. Other important aspects that have emerged since 2010 include findings of stroke risk in paroxysmal AF patients, prediction for AF-associated stroke and haemorrhage, risk-benefit considerations concerning oral anticoagulation in patients with chronic kidney disease and risk-benefit considerations with use of antiplatelet agents, alone, combined, or in combination with oral anticoagulants in AF patients. Canadian AF guidelines were updated earlier this year, incorporating the new evidence. Also, in 2012, the American College of Chest Physicians released guidelines for the antithrombotic therapy of AF. Similar updates of the ESC and the AHA/ACC guidelines are expected, which will include new findings from the cardiovascular congresses over the upcoming months. Additionally, also in 2012, the Heart Rhythm Society, the European Heart Rhythm Association and the European Cardiac Arrhythmia Society (HRS/EHRA/ECAS) released a consensus statement on the interventional treatment of AF. The following short review summarises these new data and furthermore highlights our current clinical practice.
\end{abstract}

\section{Keywords}

European Society of Cardiology, American Heart Association, American College of Cardiology, guidelines, atrial fibrillation, stroke prevention, rivaroxaban, apixaban, anticoagulation

Disclosure: Andreas Müssigbrodt has no conflicts of interest to declare. Gerhard Hindricks has received honoraria for lectures from Biosense, Sterotaxis, St Jude Medical and Biotronik, and is a member of the advisory board/consultant for Biosense, Sterotaxis, St Jude Medical, Biotronik and Cyberheart.

Received: 5 May 2012 Accepted: 20 May 2012 Citation: European Cardiology, 2012;8(3):174-8

Correspondence: Andreas Müssigbrodt, Leipzig University, Heart Center, Department of Electrophysiology, Strümpellstrasse 39, 04289 Leipzig, Germany. E: andreas.muessigbrodt@gmail.com

\section{Role of Risk Stratification Scores for the Prevention of Stroke}

Humans older than 40 years carry a $25 \%$ lifetime risk of suffering from atrial fibrillation (AF). ${ }^{1}$ The incidence of strokes attributable to AF increases from $1.5 \%$ at age 50-59 years to $23.5 \%$ at age $80-89$ years. ${ }^{2}$ Current American College of Cardiology/American Heart Association (ACC/AHA) and Canadian Cardiovascular Society (CCS) guidelines recommend the application of the Congestive heart failure, Hypertension, Age $>75$, Diabetes mellitus, and prior Stroke or transient ischaemic attack $\left(\mathrm{CHADS}_{2}\right.$ )score, whereas the $\mathrm{CHA}_{2} \mathrm{DS}_{2}$-VASC (Vascular disease, Age 65 to 74 Years, Sex category) score is recommended by the European guidelines as a stratification score for the risk of stroke and thromboembolism (TE). ${ }^{3-6}$ The $^{-\mathrm{CHDS}_{2}}$ score is easier to apply and better validated than the more recent $\mathrm{CHA}_{2} \mathrm{DS}_{2}$-VASC score. In contrast to the $\mathrm{CHADS}_{2}$ score, the $\mathrm{CHA}_{2} \mathrm{DS}_{2}$-VASC score allows more precise risk stratification within the population at a low-risk for stroke and TE. ${ }^{7.8}$ Recently published results showed that the $\mathrm{CHA}_{2} \mathrm{DS}_{2}$-VASc score gives a more accurate prediction of risks than the $\mathrm{CHADS}_{2}$ score, with risk increasing with each point on the $\mathrm{CHA}_{2} \mathrm{DS}_{2}$-VASc scale. Of particular note is the lowest risk patients on the $\mathrm{CHADS}_{2}$ scale, with a score of 0 , were not all actually low-risk, with one-year event rates ranging from
$0.84\left(\mathrm{CHA}_{2} \mathrm{DS}_{2}\right.$-VASC score $\left.=0\right)$ to $3.20\left(\mathrm{CHA}_{2} \mathrm{DS}_{2}-\mathrm{VASC} \text { score }=3\right)^{9}{ }^{9}$ However, there is a lack of evidence that the $\mathrm{CHA}_{2} \mathrm{DS}_{2}$-VASC is superior to the $\mathrm{CHADS}_{2}$ score in impacting clinical decisions regarding the choice of antithrombotic therapy and affecting the clinical outcome.

The 2012 CCS guidelines suggest that patients at low risk of stroke $\left(\mathrm{CHADS}_{2}=0\right)$ should consider additional risk factors for stroke (including age 65-74 years, female sex and presence of vascular disease). Thus, the additional risk factors that are incorporated in the $\mathrm{CHA}_{2} \mathrm{DS}_{2}$-VASC score are taken into account. For patients at low risk $\left(\mathrm{CHADS}_{2}=0\right)$ and intermediate risk $\left(\mathrm{CHADS}_{2}=1\right)$ the use of additional risk factors will help to improve the prevention of stroke and transient ischaemic attack (TIA). Since there is a significant percentage of patients with $\mathrm{CHADS}_{2}=0$ or 1 , suffering from stroke and TE, further risk stratification is justified. Retrospective analysis of embolic strokes underlines the advantage of the use of additional risk factors, such as the $\mathrm{CHA}_{2} \mathrm{DS}_{2}$-VASC score. $7,9,10$

We use the $\mathrm{CHA}_{2} \mathrm{DS}_{2}$-VASC score to identify patients at a very low-risk of stroke, for whom oral anticoagulants do not reduce the risk of stroke significantly. 


\section{Role of Risk Stratification Scores for the Prevention of Bleeding}

Due to the concern of bleeding, many high-risk patients are undertreated with oral anticoagulants (OAC). The 2012 CCS guidelines ${ }^{4}$ recommend the HAS-BLED (Hypertension, Abnormal Renal/Liver Function, Stroke, Bleeding History or Predisposition, Labile international normalised ratio [INR], Elderly, Drugs/Alcohol Concomitantly) score to be applied to all patients with AF. This score is already included in the 2010 European Society of Cardiology (ESC) guidelines, ${ }^{3}$ mainly to assist for the decision-making between the higher and lower dosage for dabigatran. However, clinical consequences in case of an increased HAS-BLED score are difficult to draw. As a matter of fact, an increased HAS-BLED score always goes along with an increased $\mathrm{CHADS}_{2}$ or $\mathrm{CHA}_{2} \mathrm{DS}_{2}$-VASC score. For most patients the long-term consequences of a stroke outweigh the long-term consequences of bleeding. Moreover, prospective validation of the HAS-BLED score is still lacking. The Birmingham Atrial Fibrillation Treatment of the Aged (BAFTA) trial has shown that the use of aspirin in elderly patients does not reduce the risk of major bleedings compared with vitamin $\mathrm{K}$ antagonists (VKA), whereas significantly more strokes and TE occur. ${ }^{11}$ The increased risk of bleeding when multiple anticoagulants are administered is well-known. Major bleeding is an important contributor to morbidity and mortality. ${ }^{12}$

In our clinical practice, we try to avoid triple or quadruple anticoagulation or to keep it as short as possible. Difficult cases, like patients with mechanical valves under bridging therapy with heparin and VKA and need for dual antiplatelet therapy deserve longer hospitalisation and close monitoring of anticoagulation. We primarily estimate the risk of bleeding based on clinical picture, including history of haemorrhage and renal function, rather than based on the HAS-BLED score.

\section{Role of New Oral Anticoagulants in the Prevention of Stroke}

As recent trials have shown, novel OAC offer an improved safety profile, compared with VKA. ${ }^{13-15}$ Consequently, the 2012 Canadian guidelines suggest that when OAC therapy is indicated, most patients should receive dabigatran, rivaroxaban, or apixaban (once approved) in preference to warfarin. This recommendation places a relatively high value on comparisons with warfarin showing that dabigatran and apixaban have greater efficacy and that rivaroxaban has similar efficacy for stroke prevention. Dabigatran and rivaroxaban have no more major bleeding and apixaban has less; all three new OACs have less intracranial haemorrhage and are simpler to use. The preference for one of the novel OACs over VKA is less marked amongst patients already receiving warfarin with stable INRs and no bleeding complications. The obvious advantages of the new OAC in recent clinical trials are given a higher value in the Canadian guidelines than the relatively short clinical experience with them. The recommendation places less value on the following features of VKA: long experience with clinical use; availability of a specific antidote; and a simple and standardised test for intensity of anticoagulant effect. The concern of increased incidence of myocardial infarctions (MI) under treatment with dabigatran has been relieved by newer meta-analysis, including data from the Randomized Evaluation of Long term anticoagulant therapY (RE-LY) trial. ${ }^{4}$ Moreover, in the Rivaroxaban Once daily oral direct factor Xa inhibition Compared with vitamin $\mathrm{K}$ antagonist for prevention of stroke and Embolism Trial in Atrial Fibrillation (ROCKET-AF), the Ml relative risk (RR) for rivaroxaban versus warfarin was 0.81 . In the Apixaban for Reduction In Stroke and Other Thromboembolic Events in Atrial Fibrillation (ARISTOTLE) trial, the MI RR for apixaban versus warfarin was $0.61 .{ }^{4}$
The 2012 CCS guidelines suggest OAC therapy for patients with a $\mathrm{CHADS}_{2}=0$ and additional risk factors (age greater than 65 or the combination of female sex and vascular disease); acetylsalicylic acid (ASA) (75-325 mg/day) for patients at lower risk within this category (female sex or vascular disease); and no antithrombotic therapy for those patients at lowest risk in this category (no additional risk factors).

These recommendations go along with the recommendations from the 2010 ESC guidelines. The 2012 CCS guidelines recommend regular assessment of renal function in patients receiving OAC. This is of particular importance because novel OAC can accumulate when renal function is impaired in chronic kidney disease (CKD). Renal function can deteriorate by intermittent dosage increase of diuretics, a common treatment in patients with heart failure. Moreover, patients on dialysis do not benefit from systematic anticoagulation, therefore, careful choice of anticoagulation has to be applied in patients with CKD. According to the CCS guidelines, for patients with an estimated glomerular filtration rate (eGFR) $>30 \mathrm{ml} / \mathrm{min}$, antithrombotic therapy should be used according to their $\mathrm{CHADS}_{2}$ score as for patients with normal renal function. Patients with an eGFR $15-30 \mathrm{ml} / \mathrm{min}$ (not on dialysis) should be treated preferably with VKA. Despite the fact that most patients on dialysis do not benefit from anticoagulation for stroke prevention, anticoagulation can be prescribed for some of those patients after an individual risk-benefit assessment. ${ }^{16}$

As a result of the significant renal excretion of dabigatran ( $80 \%$ renal excretion), we think it is prudent to consider dosage adaption early on at an eGFR below $50 \mathrm{ml} / \mathrm{min}$. In many countries dabigatran is offered in two different dosages. We use $110 \mathrm{mg}$ dabigatran in patients with impaired renal function and eGFR below $50 \mathrm{ml} / \mathrm{min}$ and above $30 \mathrm{ml} / \mathrm{min}$. Rivaroxaban (66 \% renal excretion) also requires reduction of dosage from 20 to $15 \mathrm{mg} / \mathrm{d}$ for patients with CKD and eGFR $<30 \mathrm{ml} / \mathrm{min}$ but $>15 \mathrm{ml} / \mathrm{min}$. Abcixaban has a lower renal excretion (25\% renal excretion). Thus, abcixaban or VKA might be considered first-line treatment for patients with CKD.

In our clinical practice we use $\mathrm{CHA}_{2} \mathrm{DS}_{2}$-VASC score and individual risk assessment for the decision concerning anticoagulation. Individual risk assessment should include the steady increase of risk with ageing and the history of strokes and TE in the family of the patient. We treat newly diagnosed AF with one of the novel OAC in a great number of cases. For patients with stable INR under VKA we recommend continuation of the VKA. From our point of view, the decision to prescribe OAC should be driven more by the patient's risk of stroke than by the risk of bleeding. For patients with increased risk of bleeding a change from VKAs to new generation OAC with decreased risk of bleeding should be considered first, rather than discontinuing or not treatment with OAC.

\section{Role of Catheter Ablation in the Prevention of Stroke}

Catheter ablation for AF has the potential to provide definitive therapy and as such, could potentially obviate the need for chronic anticoagulation in patients who successfully maintain sinus rhythm after the procedure. However, recurrence of AF occurs in about $30 \%$ of patients. AF recurrences after catheter ablation are usually less symptomatic.

Therefore, current Heart Rhythm Society, European Heart Rhythm Association and European Cardiac Arrhythmia Society (HRS/EHRA/ECAS) guidelines ${ }^{17}$ recommend continuing OAC for at least two months following an AF ablation procedure. Thereafter, the decision regarding the continuation of OAC agents more than two months following 
ablation should be based on the patient's risk factors for stroke and not on the presence or type of AF. The discontinuation of systemic anticoagulation therapy post-ablation is not recommended in patients who are at increased risk of stroke.

In contrast to the current HRS/EHRA/ECAS guidelines, the long-term outcome of 327 patients after ablation of AF, withholding anticoagulation in patients with sinus rhythm was examined in one recent study. Patients with a $\mathrm{CHADS}_{2}$ score of 2 and 3 accounted for $68.8 \%$ of the cohort. After a follow-up of four years (range, 1.6-8.0 years), $82 \%$ remained AF free. All patients were kept on antiplatelet drugs. No symptomatic ischaemic cerebrovascular events were detected despite interruption of anticoagulation in 298 (91\%) patients and antiarrhythmic drugs in 293 (89 \%) patients. Major adverse events were haemorrhagic strokes in three patients who continued to receive anticoagulation. ${ }^{18}$

These impressive data should be carefully considered and, for now, not lead to a more liberal behaviour in withholding consequent anticoagulation outside from clinical studies. One finding that also deserves to be taken into account before considering to discontinue anticoagulation in patients after successful AF ablation, is the potential of the $\mathrm{CHADS}_{2}$ score to predict strokes in patients with coronary heart disease and unknown AF. ${ }^{19}$

However, to discover whether successful AF ablation is able to reduce the risk of stroke, a tight follow-up is desirable. Implantable loop recorders (ILRS) have a high sensitivity to detect AF. The overall accuracy of ILR of detecting AF is $98.5 \%{ }^{20}$ Further studies issuing the withdrawal of anticoagulation after AF ablation should consider the use of ILRS or other implanted devices, such as a pacemaker or implantable cardioverter defibrillator (ICD), possibly in connection with remote control technology. Due to the still lacking evidence of the value of interventional treatment for AF from trials with hard endpoint parameters, the significance of two ongoing trials needs to be stressed.

The Catheter Ablation versus Antiarrhythmic Drug Therapy for Atrial Fibrillation (CABANA) trial (NCT00911508) is designed to enrol a sufficiently large number of patients and continue for a long enough period of time to determine if there is a mortality benefit for patients undergoing catheter ablation of AF. The CABANA study will also investigate other outcomes of AF ablation and drug therapy including cardiovascular death, occurrence of disabling stroke, serious bleeding and cardiac arrest. Rather than comparing any specific drug therapy against an individual ablative intervention, this trial will compare early aggressive rhythm control versus usual care in patients with AF less than one year. ${ }^{17}$

The Early treatment of Atrial fibrillation for Stroke prevention Trial (EAST) (NCT01288352) is also designed to examine serious endpoints, including mortality and other major morbidities. EAST tests whether early rhythm control therapy can improve hard outcome parameters in AF patients compared with usual care alone. Unlike CABANA, EAST does not compare AF ablation to antiarrhythmic drug therapy, but rather tests whether an early application of rhythm control therapy encompassing ablation, antiarrhythmic drugs or both, can prevent strokes, cardiovascular deaths, acute coronary syndromes, or decompensated heart failure compared with common therapy. ${ }^{17}$

As an interesting side note, the principal investigators of both trials will collaborate to analyse pooled data. Both trials will help to answer the open question whether AF ablation offers benefit beyond quality of life aspects.
As a result of the recommendation to continue anticoagulation in most patients, even after successful ablation, the potential risk reduction of stroke after ablation of AF will not be issued by those two trials.

Embolism of air or thrombus is a potentially life-threatening complication of interventional treatment of AF. The incidence of peri-interventional TE is reported to be between 0 and $7 \%$. Thromboembolic events typically occur within 24 hours of the ablation procedure with an increased risk during the first two weeks following ablation. Thrombi formation can occur on or within sheaths or ablation catheters positioned within the left atrium (LA), char formation, dislocation of a pre-existing thrombus; and stunning may occur after cardioversion.

Thromboembolic complications may be reduced by precise pre-procedural imaging to rule out thrombi. A consequent anticoagulation protocol with constant heparinised flush through all long sheaths, and careful application of radio frequency (RF) energy may be applied to minimise the risk of char formation. Symptomatic thromboembolic events should prompt treatment to reduce sequelae. However, the value of conservative treatment of stroke and TE versus more aggressive treatment with local or systemic thrombolysis is still a matter of debate, as well as the significance of silent embolism.

Silent cerebral embolism is defined as an occlusion of a blood vessel in the brain due to an embolus that does not result in any acute clinical symptoms and is therefore 'silent'. Silent cerebral emboli can result from a thrombus, air, gas, tissue or fat. Recently, several centres have reported that diffusion-weighted magnetic resonance imaging (DW-MRI) can detect new acute lesions created by emboli following 7-38 \% of AF ablation procedures. The incidence seems to be mainly influenced by the catheter used for ablation and has been reported to be highest with the use of non-irrigated circumferential multielectrode ablation catheters. The clinical significance of silent cerebral embolism still needs to be determined. However, in the absence of a proven negative impact on neurological function in patients with acute DW-MRI lesions, a reduction in the incidence of silent cerebral embolism is desirable. Factors that are now being examined in ongoing studies include the impact of silent cerebral embolism on neurocognitive function, the influence of the anticoagulation regime, and the influence of continuous flushing of sheaths and an increased vigilance for air embolism. ${ }^{17}$

In our opinion, there is no sufficient evidence to date, which shows that stroke and TE can be confidently prevented by ablation of AF. There is, however, a measurable risk of clinical and silent strokes and TE associated with $\mathrm{AF}$ ablation. As a result of the risk of asymptomatic recurrence of $\mathrm{AF}$ or atrial flutter it is prudent to continue anticoagulation after AF ablation, based on the thromboembolic risk. Exceptions to this rule can be individually considered only in few patients with low thromboembolic risk. These aspects should be discussed with all patients prior AF ablation.

\section{Role of Left Atrial Appendage Closure in the Prevention of Stroke}

The left atrial appendage (LAA) is considered the main source of thrombi inducing TE and strokes in patients suffering from AF. ${ }^{21}$ This is supported by the fact that transoesophageal echocardiography detects most thrombi in the LAA. ${ }^{22}$ Low stroke rates are reported in patients in whom the LAA has been surgically removed. ${ }^{23}$

Thus, surgical excision or stapling of the LAA is widely performed as a concomitant procedure during open heart surgery. ${ }^{24}$ Although frequently 
performed, there is no conclusive evidence that surgical LAA excision or occlusion reduces strokes in AF patients, due to a lack of large controlled trials with systematic follow-up. In addition, no conclusive data on the optimal surgical technique to perform LAA closure are available.

Thus, several interventional techniques have been developed for occlusion of the LAA orifice to reduce the stroke risk. ${ }^{25-27}$ Such devices could provide an alternative treatment to oral anticoagulation for AF patients with a high stroke risk but contraindications to chronic oral anticoagulation. If the efficiency of LAA closure could be conclusively shown, device-based therapy could potentially replace long-term oral anticoagulation.

Currently two devices for LAA occlusion are available for clinical use in Europe: the Watchman ${ }^{\circledR}$ and the Amplatzer ${ }^{\mathrm{TM}}$ Cardiac Plug. ${ }^{25-27}$ Their evaluation is ongoing in controlled trials: The WATCHMAN left atrial appendage system for embolic PROTECTion in patients with Atrial Fibrillation (PROTECT AF) trial randomised 707 eligible patients to percutaneous closure of the LAA using the Watchman device $(n=463)$, or to oral anticoagulation (INR range $2-3$; control, $n=244$ ). ${ }^{28}$ Patients randomised to LAA occlusion were treated with OAC for 45 days followed by dual platelet inhibition for six months and aspirin alone as chronic therapy. The primary efficacy event rate (composite endpoint of stroke, cardiovascular death and systemic embolism) of the LAA occlusion group was not inferior compared with patients treated with oral anticoagulation. There was a high number of adverse safety events in the intervention group mainly due to peri-procedural complications. Most of the adverse safety events in the device group occurred early during the trial, most likely due to the operator's learning curve. A registry (Continued Access to PROTECT AF [CAP]) follows patient outcomes beyond the end of enrolment. ${ }^{29}$ A second randomised trial, Evaluation of the WATCHMAN LAA Closure Device in Patients With Atrial Fibrillation Versus Long Term Warfarin Therapy (PREVAIL), (NCT01182441) is currently under way.

In a feasibility and safety study LAA occlusion with the Amplatzer Cardiac Plug was attempted in 137 of 143 patients, and successfully performed in 132 (96 \%). ${ }^{27}$ There were serious complications in 10 (7\%) patients. A randomised prospective study with the device is currently ongoing (Amplatzer Cardiac Plug Trial, NCT01118299).
Overall, the total number of patients enrolled in trials being treated with the two devices currently available in Europe is, to our knowledge, $n=1,896$, and the stroke rate after successful device implantation is 2-3 \% per year. Periprocedureral complications, mostly consisting of pericardial effusions, occurred overall in $5 \%$, with a tendency to decrease with progressing learning curve. ${ }^{28}$

Even on optimal anticoagulation, there is a considerable rate of residual strokes in AF patients, amounting to $1.4 \%$ per year on average in contemporary controlled trials with novel OAC. ${ }^{13-15}$ Thus, there is a need for improved stroke prevention for patients who experience a stroke on optimal oral anticoagulation. ${ }^{30}$ Those patients are potential candidates for a combination therapy consisting of LAA closure and oral anticoagulation. To date, clinical data on these patients are lacking.

Although the concept of surgical or interventional LAA closure appears reasonable, the evidence of efficacy and safety is currently insufficient to recommend these approaches to any patients other than those in whom long-term oral anticoagulation is absolutely contraindicated. Furthermore, there are data to suggest that not all strokes in AF patients are cardioembolic or due to $A F^{31}$ suggesting that there might be a need for antithrombotic therapy in AF patients after removal or closure of the LAA. Additional, adequately powered randomised studies in patients with high stroke risk and long-term follow-up comparing interventional/surgical LAA closure with novel oral anticoagulation drugs are required for adequate assessment of such techniques.

\section{Conclusion}

New generation OAC play an important role in the prevention of stroke and TE and probably oust VKA on the long run. The role of catheter ablation in stroke prevention will be elucidated by current and upcoming trials. Those trials will mainly affect the future of interventional electrophysiology. Based on current data, surgical or interventional LAA closure might be considered in patients with a high-risk for stroke and $\mathrm{TE}$, and contraindications for long-term oral anticoagulation. Current data does not support surgical or interventional LAA closure as a co-equal alternative to oral anticoagulation therapy. New data will influence our clinical decision-making but should, however, not prevent us from an individual approach to every single patient.
1. Lloyd-Jones DM, Wang TJ, Leip EP, et al., Lifetime risk for development of atrial fibrillation: the Framingham Heart Study, Circulation, 2004:110:1042-6.

2. Wolf PA, Abbott RD, Kannel WB, Atrial fibrillation as an independent risk factor for stroke: the Framingham Study, Stroke, 1991;22:983-8.

3. Camm AJ, Kirchhof P, Lip GY, et al., Guidelines for the management of atrial fibrillation: the Task Force for the Management of Atrial Fibrillation of the European Society of Cardiology (ESC), Eur Heart J, 2010;31:2369-429.

4. Skanes AC, Healey JS, Cairns JA, et al., Focused 2012 update of the Canadian Cardiovascular Society atrial fibrillation guidelines: recommendations for stroke prevention and guidelines: recommendations for stroke prevention a
rate/rhythm control, Can I Cardiol, 2012;28:125-36.

5. Fuster V, Ryden LE, Cannom DS, et al., ACC/AHA/ESC 2006 Guidelines for the Management of Patients with Atrial Fibrillation: a report of the American College of Cardiology/American Heart Association Task Force on Practice Guidelines and the European Society of Cardiology Committee for Practice Guidelines (Writing Committee to Revise the 2001 Guidelines for the Management of Patients With Atrial Fibrillation): developed in collaboration with the European Heart Rhythm Association and the Heart Rhythm Society, Circulation, 2006;114:257-354.

6. Wann LS, Curtis AB, Ellenbogen KA, et al., 2011 ACCF/AHA/HRS focused update on the management of patients with atrial fibrillation (update on Dabigatran): a report of the American College of Cardiology Foundation/American Heart Association Task Force on practice guidelines, Circulation, 2011;123:1144-50.

7. Lip GY, Nieuwlaat R, Pisters $R$, et al., Refining clinical risk stratification for predicting stroke and thromboembolism in atrial fibrillation using a novel risk factor-based approach: the euro heart survey on atrial fibrillation, Chest, 2010:137:263-72.

8. Olesen JB, Lip GY, Hansen ML, et al., Validation of risk stratification schemes for predicting stroke and thromboembolism in patients with atrial fibrillation: nationwide cohort study, BMJ, 2011;342:d124.

9. Olesen JB, Torp-Pedersen C, Hansen ML, Lip GY, The value of the CHA2DS2-VASC score for refining stroke risk stratification in patients with atrial fibrillation with a CHADS2 score 0-1: A nationwide cohort study, Thromb Haemost, 2012;107(6):1172-9.

10. Pieri A, Lopes TO, Gabbai AA, Stratification with CHA2DS2-VASC score is better than CHADS2 score in reducing ischemic stroke risk in patients with atrial fibrillation, Int J Stroke, 2011;6:466.

11. Mant J, Hobbs FD, Fletcher $\mathrm{K}$, et al.,Warfarin versus aspirin for stroke prevention in an elderly community population with atrial fibrillation (the Birmingham Atrial Fibrillation Treatment of the Aged Study, BAFTA): a randomised controlled trial, Lancet, 2007;370:493-503.

12. Alberts MJ, Bhatt DL, Smith SC, et al., Risk factors and outcomes for patients with vascular disease and serious bleeding events, Heart, 2011;97:1507-12.

13. Connolly SJ, Ezekowitz MD, Yusuf S, et al., Dabigatran versus warfarin in patients with atrial fibrillation, N Eng/ J Med, 2009:361:1139-51.

14. Patel MR, Mahaffey KW, Garg J, et al., Rivaroxaban versus warfarin in nonvalvular atrial fibrillation, $N$ Engl I Med 2011;365:883-91

15. Granger $\mathrm{CB}$, Alexander $\mathrm{JH}, \mathrm{McMurray} \mathrm{JJ}$, et al., Apixaban versus warfarin in patients with atrial fibrillation, $N$ Eng/ I Med 2011;365:981-92

16. Chan KE, Lazarus JM, Thadhani R, Hakim RM, Anticoagulant and antiplatelet usage associates with mortality among hemodialysis patients, J Am Soc Nephrol, 2009;20:872-81.

17. Calkins H, Kuck KH, Cappato R, et al., 2012 HRS/EHRA/ECAS Expert Consensus Statement on Catheter and Surgical Ablation of Atrial Fibrillation: Recommendations for Patient Selection, Procedural Techniques, Patient Management and Follow-up, Definitions, Endpoints, and Research Trial Design: A report of the Heart Rhythm Society (HRS) Task Force on Catheter and Surgical Ablation of Atrial Fibrillation. Developed in partnership with the European Heart Rhythm Association (EHRA), a registered branch of the European Society of Cardiology (ESC) and the European Cardiac Arrhythmia Society (ECAS); and in collaboration with the Ar hyth ila Society (ECAS), and in collaboration with the Association (AHA), the Asia Pacific Heart Rhythm Society (APHRS), and the Society of Thoracic Surgeons (STS). (APHRS), and the Society of Thoracic Surgeons (STS).
Endorsed by the governing bodies of the American College of Cardiology Foundation, the American Heart Association, the European Cardiac Arrhythmia Society, the European Heart Rhythm Association, the Society of Thoracic Surgeons, the Asia Pacific Heart Rhythm Society, and the Heart Rhythm Society, Heart Rhythm, 2012:9:632-96.

18. Saad $E B$, d'Avila $A$ costa IP, et al very low risk of thromboembolic events in patients undergoing successful catheter ablation of atrial fibrillation with a CHADS2 score $</=3$ : a long-term outcome study, Circ Arrhythm Electrophysiol, $</=3$ : a long-te

19. Welles CC, Whooley MA, Na B, et al., The CHADS2 score predicts ischemic stroke in the absence of atrial fibrillation among subjects with coronary heart disease: data from the Heart and Soul Study, Am Heart J, 2011;162:555-61.

20. Hindricks G, Pokushalov E, Urban L, et al., Performance of a new leadless implantable cardiac monitor in detecting and 
quantifying atrial fibrillation: Results of the XPECT trial, Circ Arrhythm Electrophysiol, 2010;3:141-7.

21. Ferro JM, Cardioembolic stroke: an update, Lancet Neurol 2003;2(3):177-88.

22. Klein AL, Grimm RA, Murray RD, et al., Use of transesophageal echocardiography to guide cardioversion in patients with atrial fibrillation, N Eng/ / Med, 2001:344:1411-20.

23. Healey JS, Crystal E, Lamy A, et al., Left Atrial Appendage Occlusion Study (LAAOS): results of a randomized controlled pilot study of left atrial appendage occlusion during coronary bypass surgery in patients at risk for stroke, Am Heart J, 2005;150:288-93.

24. Whitlock RP, Healey JS, Connolly SJ, Left atrial appendage occlusion does not eliminate the need for warfarin, Circulation, 2009:120:1927-32.
25. Bayard YL, Omran H, Neuzil P, et al., PLAATO (Percutaneous Left Atrial Appendage Transcatheter Occlusion) for prevention of cardioembolic stroke in non-anticoagulation eligible atrial fibrillation patients: results from the European PLAATO study, Eurolntervention, 2010;6:220-6

26. Sick PB, Schuler $G$, Hauptmann $K E$, et al., Initial worldwide experience with the WATCHMAN left atrial appendage system for stroke prevention in atrial fibrillation I Am Coll Cardiol, 2007;49:1490-5.

27. Park JW, Bethencourt A, Sievert H, et al., Left atrial appendage closure with Amplatzer cardiac plug in atrial fibrillation: initial European experience,

Catheter Cardiovasc Interv, 2011;77:700-6.

28. Holmes DR, Reddy VY, Turi ZG, et al., Percutaneous closure of the left atrial appendage versus warfarin therapy for prevention of stroke in patients with atrial fibrillation: a randomised non-inferiority trial, Lancet, 2009:374:534-42.

29. Reddy VY, Holmes D, Doshi SK, et al., Safety of

percutaneous left atrial appendage closure: results from the Watchman Left Atrial Appendage System for Embolic Protection in Patients with AF (PROTECT AF) clinical trial and the Continued Access Registry, Circulation,

30. Kirchhof P, Lip GY, Van Gelder IC, et al., Comprehensive risk reduction in patients with atrial fibrillation: emerging diagnostic and therapeutic options--a report from the 3rd Atrial Fibrillation Competence NETwork/European Heart Rhythm Association consensus conference, Europace, 2012:14:8-27.

31. Lip GY, Lim HS, Atrial fibrillation and stroke prevention, Lancet Neurol, 2007:6:981-93. 